\title{
The $\beta$-Amyloid Precursor Protein of Alzheimer's Disease Enhances Neuron Viability and Modulates Neuronal Polarity
}

\author{
Ruth G. Perez, ${ }^{1,3}$ Hui Zheng, ${ }^{4}$ Lex H. T. Van der Ploeg, ${ }^{4}$ and Edward H. Koo ${ }^{2,3}$ \\ Departments of ${ }^{1}$ Neurology and ${ }^{2}$ Pathology, Harvard Medical School, Boston, Massachusetts 02115, ${ }^{3}$ Center for

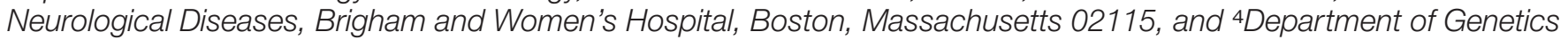 \\ and Molecular Biology, Merck Research Laboratories, Rahway, New Jersey 07065
}

\begin{abstract}
$\beta$-Amyloid precursor protein $(\beta \mathrm{PP})$ can reside at neuron and glial cell surfaces or undergo proteolytic processing into secreted fragments. Although $\beta P P$ has been studied extensively, its precise physiological role is unknown. A line of transgenic knock-out mice selectively deficient in $\beta P P$ survive and breed but exhibit motor dysfunction and brain gliosis, consistent with a physiological role for $\beta$ PP in neuron development. To elucidate these functions, we cultured hippocampal neurons from wild-type and $\beta$ PP-deficient mice and compared their ability to attach, survive, and develop neurites. We found that hippocampal neurons from $\beta$ PP-deficient mice had diminished viability and retarded neurite development. We also compared the effects of $\beta P P$ secretory products, released from wild-type as-
\end{abstract}

trocytes, on process outgrowth from wild-type and $\beta \mathrm{PP}$ deficient hippocampal neurons. Outgrowth was enhanced at $1 \mathrm{~d}$ in the presence of wild-type astrocytes, as compared with $\beta P P$-deficient astrocytes. However, by $3 \mathrm{~d}$, neurons had shorter axons but more minor processes with more branching when cocultured with wild-type astrocytes, as compared with $\beta \mathrm{PP}$ deficient astrocytes. Our data demonstrate that cell-associated neuronal $\beta$ PP contributes to neuron viability, axonogenesis, and arborization and that $\beta \mathrm{PP}$ secretory products modulate axon growth, dendrite branching, and dendrite numbers.

Key words: arborization; astrocytes; axonogenesis; $A \beta ; \beta P P$; $\beta P P_{s}$; knock-out mice; neurite outgrowth; neuron survival
$\beta$-Amyloid precursor protein ( $\beta \mathrm{PP})$ is a type I integral membrane protein that shares homology with glycosylated membrane receptors (Kang et al., 1987) and is present on the surface of neuronal and glial cells (Shivers et al., 1988; Breen et al., 1991; Yamazaki et al., 1997). $\beta \mathrm{PP}$ also can be processed proteolytically by a number of enzymes referred to as "secretases" into soluble $\beta P P$ fragments including $\beta \mathrm{PP}_{\mathrm{s}}$, the large $\mathrm{N}$-terminal secreted fragment, and $\mathrm{A} \beta$, the major protein component of senile plaques in Alzheimer's disease (for review, see Selkoe, 1994). Because A $\beta$ deposition may be central to Alzheimer's disease pathogenesis, much research has focused on the generation of this small peptide from its full-length precursor. $\beta \mathrm{PP}$ is expressed abundantly by embryonic neurons and astrocytes (Ferreira et al., 1993; Moya et al., 1994; Yamazaki et al., 1995). Although $\beta P P$ is expressed normally in the CNS throughout life and has been studied for years, the precise functions of full-length cell-associated $\beta \mathrm{PP}$ and its secreted fragments are still unclear. $\beta$ PP has been implicated in cell adhesion (Schubert et al., 1989), cell growth (Saitoh et al., 1989), neurite outgrowth (Milward et al., 1992; Qiu et al., 1995), and neuroprotection (Goodman and Mattson, 1994). One means of evaluating the role of $\beta \mathrm{PP}$ is by studying neuronal cells derived

Received July 24, 1997; revised Sept. 2, 1997; accepted Sept. 24, 1997.

This work was supported by National Institutes of Health Grants NS28121 (E.H.K.) and 5T32AG00222 (R.G.P.) and the Paul Beeson Physician Faculty Scholar in Aging Research from the American Federation for Aging Research (E.H.K.). We are grateful to Dr. A. Frankfurter for the gift of TuJ1 antibody; Drs Adriana Ferreira, Tsuneo Yamazaki, and Gloria Lee for technical advice and supportive discussions; and Drs. Willi Halfter, Deborah Watson, and Margaret Kruse for critical reading of this manuscript.

Correspondence should be addressed to Dr. Ruth G. Perez, Allegheny University of the Health Sciences, Neurosciences Research Center, 320 East North Avenue/ 10th Floor, South Tower, Pittsburgh, PA 15212-4772.

Dr. Koo's present address: Department of Neurosciences 0691, University of California, San Diego, 9500 Gilman Drive, La Jolla, CA 92093-0691.

Copyright (C) 1997 Society for Neuroscience $0270-6474 / 97 / 179407-08 \$ 05.00 / 0$ from $\beta$ PP-deficient mice (Zheng et al., 1995) and comparing them with wild-type neuronal cells. Some of the phenotypic abnormalities associated with $\beta$ PP-deficient mice (i.e., reactive gliosis in hippocampus and neocortex plus a reduced forelimb grip strength and altered locomotion) imply that $\beta \mathrm{PP}$ is important for normal CNS function. Furthermore, neuronal cells with diminished $\beta P P$ expression, whether transfected with antisense $\beta$ PP (LeBlanc et al., 1992) or treated with antisense oligonucleotides (Majocha et al., 1994; Allinquant et al., 1995), have altered process growth indicating that $\beta \mathrm{PP}$ is important to neuron development.

Although A $\beta$ can be neurotrophic (Whitson et al., 1989; Yankner et al., 1990), fibrillar A $\beta$ is toxic to neurons in vitro and may cause neuron loss in the brains of Alzheimer's patients (for review, see Yankner, 1996). Because $\beta \mathrm{PP}$ is the precursor to $\mathrm{A} \beta$, knowledge of the normal role of $\beta \mathrm{PP}$ in the nervous system may clarify our understanding of $\beta \mathrm{PP}$-associated pathology found in Alzheimer's disease and help to direct potential therapeutic strategies. To this end, hippocampal neurons from wild-type and $\beta P P-d e f i c i e n t$ mice were evaluated for differences in cell attachment, survival, and neurite outgrowth in cocultures with astrocytes from wild-type and $\beta$ PP-deficient mice. The use of astrocytes from wild-type and $\beta$ PP-deficient mice provided a unique means for measuring differences in neuron development associated with secreted factors released into the astrocyte conditioned media. Our results show the importance of both cell-associated $\beta \mathrm{PP}$ and secreted $\beta \mathrm{PP}$ products on neurite differentiation, with implications for normal brain development.

\section{MATERIALS AND METHODS}

Transgenic knock-out mice. $\beta$ PP-deficient knock-out mice, generated in the C57BL6/129 hybrid background, have been described (Zheng et al., 1995). Homozygous $\beta$ PP-deficient mice survive and breed but have 
motor dysfunction and develop brain gliosis, as compared with wild-type control mice generated in the same C57BL6/129 hybrid background. Mice used in these studies were handled in accordance with the United States Public Health Policy in Humane Care and Use of Laboratory Animals and National Institutes of Health guidelines.

Primary astrocyte cultures. Glial cultures enriched in type 1 astrocytes $(>95 \%)$ were prepared as described (Tawil et al., 1993) after dissection, using the protocol of Banker and Goslin (1991). Postnatal day 1 cortical tissues from wild-type and $\beta \mathrm{PP}$-deficient mice were dissected free of choroid plexus and meninges and dissociated after trypsinization (trypsin-EDTA; Life Technologies, Gaithersburg, MD) and trituration. Astrocytes grown in $75 \mathrm{~mm}$ tissue culture flasks with minimal essential medium (MEM), 10\% horse serum, and $0.6 \%$ glucose (glia-MEM) at $37^{\circ} \mathrm{C}$ with $5 \% \mathrm{CO}_{2}$ were confluent by $10 \mathrm{~d}$. The use of a rotary shaker eliminated other cell types. Astrocytes were plated onto $60 \mathrm{~mm}$ plastic Petri dishes to form supportive monolayers.

Primary hippocampal cultures. Hippocampi were dissected from embryonic day 16 (E16) mice and prepared as described (Banker and Goslin, 1991). Briefly, dissected hippocampi were suspended in trypsinEDTA for $15 \mathrm{~min}$ at $37^{\circ} \mathrm{C}$, washed three times with calcium- and magnesium-free HBSS and triturated with a fire-polished glass pipette to dissociate cells. Hippocampal cells cultured with serum-free Neurobasal medium with B27 supplement (Life Technologies) were grown in six-well tissue culture plates or on $12 \mathrm{~mm}$ glass coverslips (Carolina Biological Supply, Burlington, NC) that were pretreated with poly-L-lysine hydrobromide ( $1 \mathrm{mg} / \mathrm{ml}$; Sigma, St. Louis, MO) in $0.1 \mathrm{M}$ borate buffer, $\mathrm{pH} 8.5$. Hippocampal cultures in B27-supplemented defined media were plated at high cell density $(68,000-102,000$ cells/well in six-well plates or 200,000 300,000 cells $/ 60 \mathrm{~mm}$ Petri dish on coverslips). Low-density hippocampal cultures for morphometric analyses were plated at 100,000 cells/60 mm Petri dish onto poly-L-lysine-treated coverslips with paraffin dots on the plating surfaces. Cells were allowed to attach for 3-5 hr in MEM/10\% horse serum $/ 0.6 \%$ glucose at $37^{\circ} \mathrm{C}$ with $5 \% \mathrm{CO}_{2}$. Then cells plated at low density on coverslips were transferred to astrocyte monolayers in serumfree N2-supplemented MEM (Bottenstein and Sato, 1979) containing ovalbumin $(0.1 \%)$ and pyruvate $(0.01 \mathrm{mg} / \mathrm{ml})$ with neurons facing, but not in direct contact with, astrocytes.

Immunocytochemistry, antibodies, neuron attachment, and neuron survival. For immunocytochemistry, cultures were fixed for $20 \mathrm{~min}$ with prewarmed 4\% paraformaldehyde $/ 4 \%$ sucrose in PBS, washed three times with PBS, and preincubated with $10 \%$ bovine serum albumin in PBS. Primary antibodies included a monoclonal antibody raised against a class III $\beta$-tubulin (clone TuJ1; Frankfurter et al., 1986) and the anti- $\alpha$-tubulin monoclonal antibody (clone DM1A; Sigma). Secondary antibodies included anti-mouse IgG-FITC for fluorescence microscopy (Jackson ImmunoResearch, West Grove, PA) and anti-mouse IgG-HRP (Amersham, Arlington Heights, IL) for bright-field microscopy. To determine initial relative numbers of neurons, we performed TuJ1 (1:250; Ferreira and Caceres, 1992) labeling of low-density hippocampal cultures in three experiments. Total cell counts by phase-contrast analysis were compared with TuJ1-stained neurons via fluorescence microscopy. The ratio of $\mathrm{TuJ} 1$-positive cells to total cell number was determined at time 0 (3-5 hr after plating) in 30 random $0.25 \times 0.25 \mathrm{~mm}$ square microscopic fields per condition.

Neuron survival was assessed by two different measures. For highdensity neuronal cultures grown in B27-supplemented medium, cells in six-well plastic tissue culture plates were immunolabeled with DM1A $(1: 250)$ and HRP-conjugated secondary antibody (1:250), followed by $3,3^{\prime}$-diaminobenzidine reaction. Uniform grids were scratched onto the plastic with a "pin rake" (Tyler Research Instruments, Edmonton, Alberta, Canada). Neurite-bearing cells were counted in 50 random 0.25 $\mathrm{mm}^{2}$ grids, using an inverted phase microscope. For low-density neurons in coculture experiments, viability was assessed directly, as reported previously (Canoll et al., 1996), by counting the numbers of live neurons in cultures, using the Live/Dead Viability/Cytotoxicity Kit according to the manufacturer (Molecular Probes, Eugene, OR). Intact (live) cells stained green with calcein-AM, whereas dead cells stained red by ethidium dimer intercalation into DNA of cells with compromised plasma membranes. Total cell counts were obtained via phase-contrast illumination. Total cell numbers and live neuron counts were obtained for 50 nonoverlapping microscopic fields for each coculture condition in three independent experiments.

Normal hippocampal neuron development in astrocyte coculture. Cocultured hippocampal neurons derived from rats (Caceres et al., 1984) or mice (Chin et al., 1995) recapitulate some of their in vivo differentiation by establishing axonal and dendritic polarity in vitro. By $1 \mathrm{~d}$ in vitro, neurons at "stage 3" (Dotti et al., 1988) have a recognizable axon, which by definition is the single longest neurite extending from the cell body. Because the nonaxonal dendritic minor processes do not compartmentalize MAP2 protein until 3-5 d in vitro (Caceres et al., 1984), they are referred to as "minor processes."

Quantitative morphometry. Neurons plated at low density were fixed and stained with DM1A (see above). Forty neurons per coculture condition were evaluated at 1 and at $3 \mathrm{~d}$ after plating. Isolated stage 3 neurons with a distinct axon were selected at random from nonoverlapping fields, and the cell body and all processes were traced from an inverted phase microscope image projected onto a monitor via video camera. Quantitative measurements were obtained with a digitizing pad, as previously described (Brandt and Lee, 1993). Neurons were evaluated for axon outgrowth, minor process outgrowth, axon and minor process branching, minor process numbers, and cell body diameters. Axon outgrowth refers to the single longest neurite extending from the neuronal cell body plus the sum of the lengths of all small processes that emanate laterally from that single longest process. Each neuron analyzed had a single axon. Minor process outgrowth consists of the sum of the lengths of all nonaxonal processes emanating directly from the cell body, including the sum of the lengths of all small lateral processes arising from those minor processes. Total outgrowth is the sum of axonal and minor process outgrowth. Branches refers to the small processes that emanate laterally from the axon (axon branches) or minor processes (minor process branches) of each neuron. Minor process number refers to the total number of processes emanating directly from the neuronal cell body, minus one. Cell body diameters represent of the average of three diameter measures across each neural cell body.

Statistical analyses. All data were analyzed with the Instat program (GraphPad Software, San Diego, CA), using Student's $t$ tests when two sets of data were compared or ANOVA for comparisons of the four neuron/astrocyte coculture conditions. Post hoc analyses of significant ANOVA data were analyzed by the Tukey-Kramer method. All data are averages \pm SEM.

\section{RESULTS}

\section{Neurons lacking $\beta$ PP expression attach efficiently to poly-L-lysine-treated substrates but have diminished viability in vitro}

The relative numbers of neurons from wild-type and $\beta \mathrm{PP}$ deficient mice hippocampal dissections were evaluated at 3-5 hr after plating. Fixed cells were immunolabeled for neuronal class III- $\beta$-tubulin with antibody TuJ1 (described above), and both total cell number and neurons were counted. At this time point, equal numbers of neurons were stained in low-density cultures from both wild-type $(10.3 \pm 0.46$ neurons $/ 0.25 \times 0.25 \mathrm{~mm}$ square field) or $\beta$ PP-deficient (11.6 \pm 0.49 neurons $/ 0.25 \times 0.25 \mathrm{~mm}$ square field) hippocampal dissections that contained $85-95 \%$ neurons. Having determined that plating of neurons was equivalent and that both wild-type and $\beta$ PP-deficient neurons attached equally well, we next assessed neuron survival.

Neuron survival was assessed initially for high cell density cultures grown on poly-L-lysine-treated tissue culture plastic in B27-supplemented Neurobasal medium. Process outgrowth from both wild-type and $\beta$ PP-deficient neurons was slower to develop in this medium; therefore, neuron counts were performed at $3 \mathrm{~d}$ when axons were clearly developed. At $3 \mathrm{~d}$, for high-density cultures plated at two- to threefold higher cell density than the low-density cultures described above, we observed more surviving neurons in wild-type cultures $(22 \pm 0.69$ neurons $/ 0.25 \times 0.25$ $\mathrm{mm}$ square field) than in $\beta$ PP-deficient cultures $(16.1 \pm 0.71$ neurons $/ 0.25 \times 0.25 \mathrm{~mm}$ square field). This cell loss was $27 \%$ greater for $\beta \mathrm{PP}$-deficient neurons $(t=5.9 ; p<0.0001)$ than for wild-type neurons, although cultures were plated at the same density from pools with equivalent numbers of neurons. 

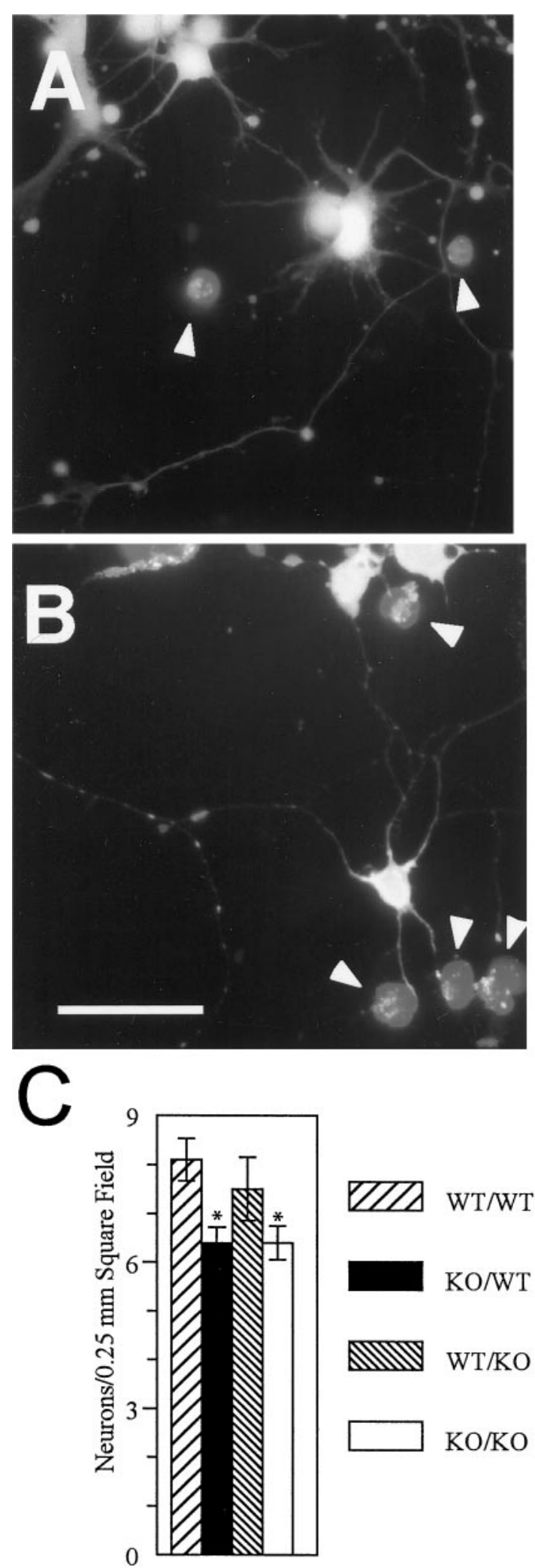

Figure 1. Live cells and dead cells in neuron/astrocyte cocultures at $3 \mathrm{~d}$ in vitro. Intact wild-type $(A)$ and $\beta$ PP-deficient $(B)$ hippocampal neurons (shown here cocultured with wild-type astrocytes) are among dead cells (at arrowheads in $A, B$ ). Live neurons appear brightly stained with calcein-AM and have well developed neurites. Dead cells, labeled by ethidium dimer, are rounded and appear faintly stained (at arrowheads in $A, B) . C, \beta P P$-deficient hippocampal cultures had significantly fewer live neurons per field whether cocultured with wild-type astrocytes or
Because neurite development was slower for neurons in the B27-supplemented medium, as compared with neurons cocultured with astrocytes (Dotti, 1988), we chose to use cocultures for subsequent experiments. Wild-type and $\beta$ PP-deficient hippocampal neurons on coverslips were cocultured above astrocytes derived from wild-type and $\beta \mathrm{PP}$-deficient mice. This produced the following four conditions: (1) wild-type neurons with wild-type astrocytes (WT/WT), (2) $\beta \mathrm{PP}$-deficient knock-out neurons with wild-type astrocytes (KO/WT), (3) wild-type neurons with $\beta \mathrm{PP}$ deficient astrocytes (WT/KO), and (4) $\beta \mathrm{PP}$-deficient neurons with $\beta \mathrm{PP}$-deficient astrocytes $(\mathrm{KO} / \mathrm{KO})$.

Neuron survival, which was less for $\beta$ PP-deficient cultures at high cell density in B27-supplemented media, again was evaluated for low-density hippocampal neurons in astrocyte cocultures. At $3 \mathrm{~d}$, wild-type hippocampal cocultures had more viable neurons (Fig. $1 A, C$ ) than $\beta$ PP-deficient hippocampal cocultures (Fig. $1 B, C)$. In these low-density cultures wild-type neurons survived similarly when cocultured with wild-type $(8.1 \pm 0.43$ neurons/ $0.25 \times 0.25 \mathrm{~mm}$ square field, Fig. $1 A, C)$ or $\beta$ PP-deficient astrocytes $(7.5 \pm 0.51$ neurons $/ 0.25 \times 0.25 \mathrm{~mm}$ square field, Fig. $1 C)$. However, $\beta$ PP-deficient neurons had equally diminished viability in coculture with wild-type $(6.36 \pm 0.31$ neurons $/ 0.25 \times 0.25 \mathrm{~mm}$ square field, Fig. $1 B)$ and $\beta$ PP-deficient astrocytes $(6.39 \pm 0.34$ neurons $/ 0.25 \times 0.25 \mathrm{~mm}$ square field, Fig. $1 C$ ), comparable to the loss observed in high-density cultures with B27-supplemented medium.

We also measured neuronal cell bodies to assess potential effects of $\beta P P$ expression on cell body attachment and spreading. Average cell body diameters were measured for 40 neurons per coculture condition in three independent experiments. Although variability was observed in all conditions, the somatic diameters were not significantly different at $1 \mathrm{~d}(\mathrm{WT} / \mathrm{WT}=15.18 \mu \mathrm{m} \pm$ $0.42 ; \mathrm{KO} / \mathrm{WT}=15.73 \mu \mathrm{m} \pm 0.39 ; \mathrm{WT} / \mathrm{KO}=15.07 \mu \mathrm{m} \pm 0.40$; and $\mathrm{KO} / \mathrm{KO}=14.67 \mu \mathrm{m} \pm 0.41)$ or at $3 \mathrm{~d}(\mathrm{WT} / \mathrm{WT}=26.4 \mu \mathrm{m} \pm$ $1.7 ; \mathrm{KO} / \mathrm{WT}=30.1 \mu \mathrm{m} \pm 2.13 ; \mathrm{WT} / \mathrm{KO}=26.64 \mu \mathrm{m} \pm 1.7$; and $\mathrm{KO} / \mathrm{KO}=27.7 \mu \mathrm{m} \pm 2.04)$, indicating that the absence of cell-associated $\beta \mathrm{PP}$ did not diminish cell body attachment and spreading.

\section{Wild-type hippocampal neurons have enhanced axon development and enhanced branching when cocultured with wild-type astrocytes for $1 \mathrm{~d}$}

All neurons measured at $1 \mathrm{~d}$ had an axon and several minor processes (for detailed descriptions of axonal and minor process outgrowth and branching, refer to Quantitative Morphometry in Materials and Methods). Wild-type neurons cocultured with wildtype astrocytes (Fig. $2 A$ ) had greater total outgrowth (Fig. $3 A$ ), as compared with neurons in the other three coculture conditions at $1 \mathrm{~d}$. This enhanced outgrowth resulted from significantly longer axons at this time (Fig. $3 A$ ). In addition, wild-type neurons cocultured with wild-type astrocytes (Fig. $2 A$ ) had more branch-

$\beta$ PP-deficient astrocytes than did wild-type hippocampal neurons cocultured with either wild-type or $\beta$ PP-deficient astrocytes in 50 random fields. $W T$, Wild-type; $K O, \beta \mathrm{PP}$-deficient knock-out. $W T / W T$, Wild-type neurons cocultured with wild-type astrocytes; $K O / W T, \beta \mathrm{PP}$-deficient neurons cocultured with wild-type astrocytes; $W T / K O$, wild-type neurons cocultured with $\beta P P$-deficient knock-out astrocytes; $K O / K O, \beta P P$-deficient neurons cocultured with $\beta$ PP-deficient astrocytes. ANOVA, $p<0.0001$. *Significantly different from WT/WT cocultures. Scale bar, $60 \mu \mathrm{m}$. 

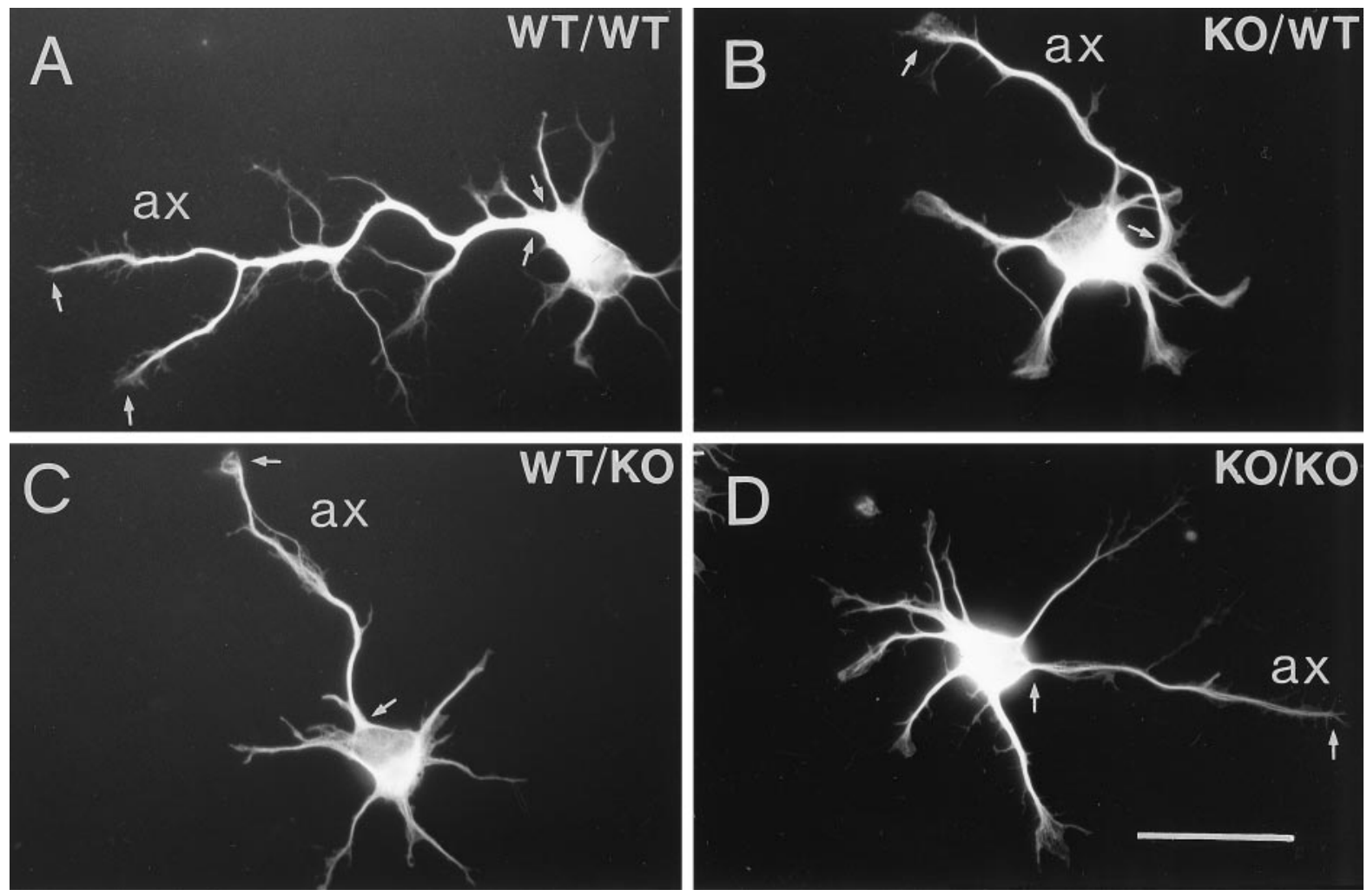

Figure 2. Stage 3 hippocampal neurons in astrocyte cocultures for $1 \mathrm{~d}$. Proximal and distal portions of each axon (ax) are marked by small arrows for a wild-type neuron cocultured with wild-type astrocytes $(A)$, a $\beta$ PP-deficient neuron cocultured with wild-type astrocytes $(B)$, a wild-type neuron cocultured with $\beta$ PP-deficient astrocytes $(C)$, and a $\beta$ PP-deficient neuron cocultured with $\beta$ PP-deficient astrocytes $(D)$. The short neurites emanating from the cell body are predendritic, minor processes. $W T / W T$, Wild-type neurons cocultured with wild-type astrocytes; $K O / W T$, $\beta$ PP-deficient neurons cocultured with wild-type astrocytes; $W T / K O$, wild-type neurons cocultured with $\beta \mathrm{PP}$-deficient knock-out astrocytes; $K O / K O$, $\beta \mathrm{PP}$-deficient neurons cocultured with $\beta$ PP-deficient astrocytes. Scale bar, $25 \mu \mathrm{m}$.

ing of axons and minor processes than did neurons in the other three coculture conditions (Figs. 2, 3B). When wild-type hippocampal neurons were cocultured with $\beta \mathrm{PP}$-deficient astrocytes (Fig. $2 C$ ), they had significantly shorter axons (Fig. $3 A$ ) with less axon branching (Fig. $3 B$ ), as compared with wild-type neurons cocultured with wild-type astrocytes. Although minor process outgrowth (Fig. $3 A$ ) and axon and minor process branching (Fig. $3 B$ ) were greater for wild-type neurons cocultured with wild-type astrocytes, these neurons actually had the least number of minor processes at $1 \mathrm{~d}$ (Fig. $3 C$ ).

$\beta$ PP-deficient neurons cocultured with wild-type (Fig. $2 B$ ) or $\beta$ PP-deficient astrocytes (Fig. 2D) had diminished total out-
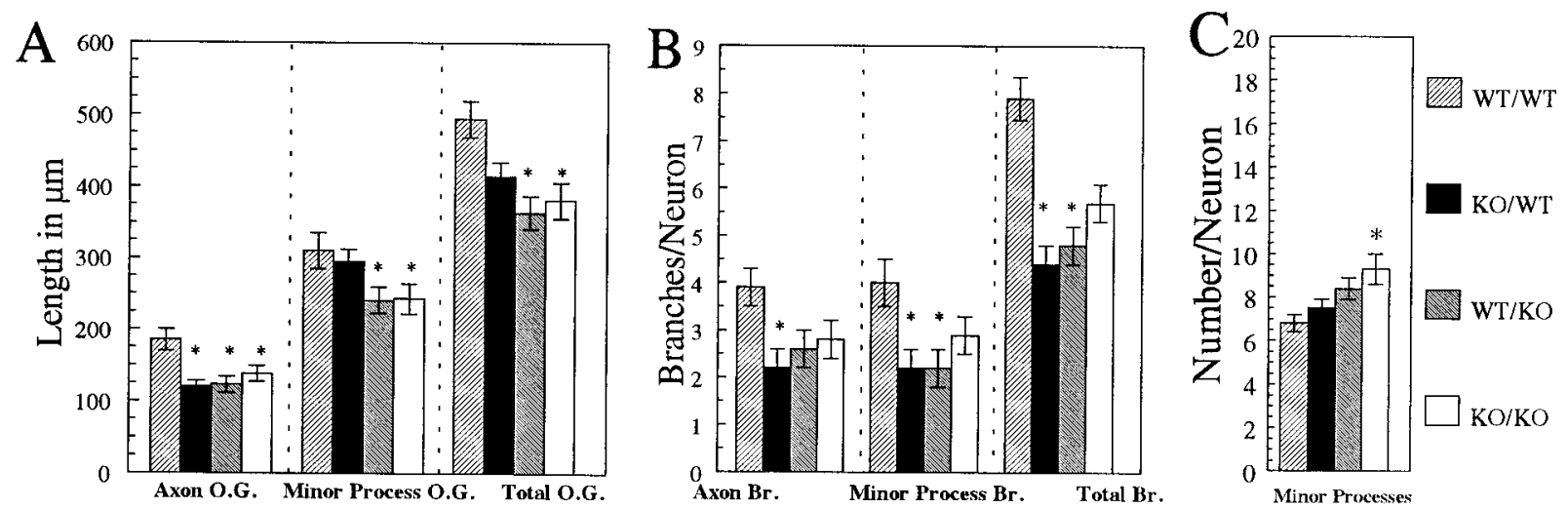

Figure 3. $\beta$ PP-related effects on neuron morphology for hippocampal neurons cocultured with wild-type and $\beta$ PP-deficient astrocytes for 1 d. $A$, Axon, minor process, and total outgrowth were greatest for wild-type neurons cocultured with wild-type astrocytes $(W T / W T)$. Significantly less axon growth was apparent for $\beta$ PP-deficient knock-out neurons in both astrocyte conditions and for wild-type neurons cocultured with $\beta$ PP-deficient astrocytes. $B$, Branching of axons and minor processes was more pronounced for neurons in WT/WT cultures. $C, \beta \mathrm{PP}$-deficient neurons, which lack cell-surface $\beta \mathrm{PP}$, had significantly more minor processes when cocultured with $\beta \mathrm{PP}$-deficient astrocytes, which do not secrete $\beta \mathrm{PP}_{\mathrm{s}}$ or $\mathrm{A} \beta . W T / W T$, Wild-type neurons cocultured with wild-type astrocytes; $K O / W T, \beta$ PP-deficient knock-out neurons with wild-type astrocytes; $W T / K O$, wild-type neurons with $\beta$ PP-deficient knock-out astrocytes; $K O / K O, \beta P P$-deficient knock-out neurons with $\beta$ PP-deficient knock-out astrocytes; $O . G$., outgrowth; $B r$., branches. Data are averages of 40 neurons \pm SEM. * Significantly different from WT/WT cocultures. Histogram legend in $C$ applies to $A-C$. 

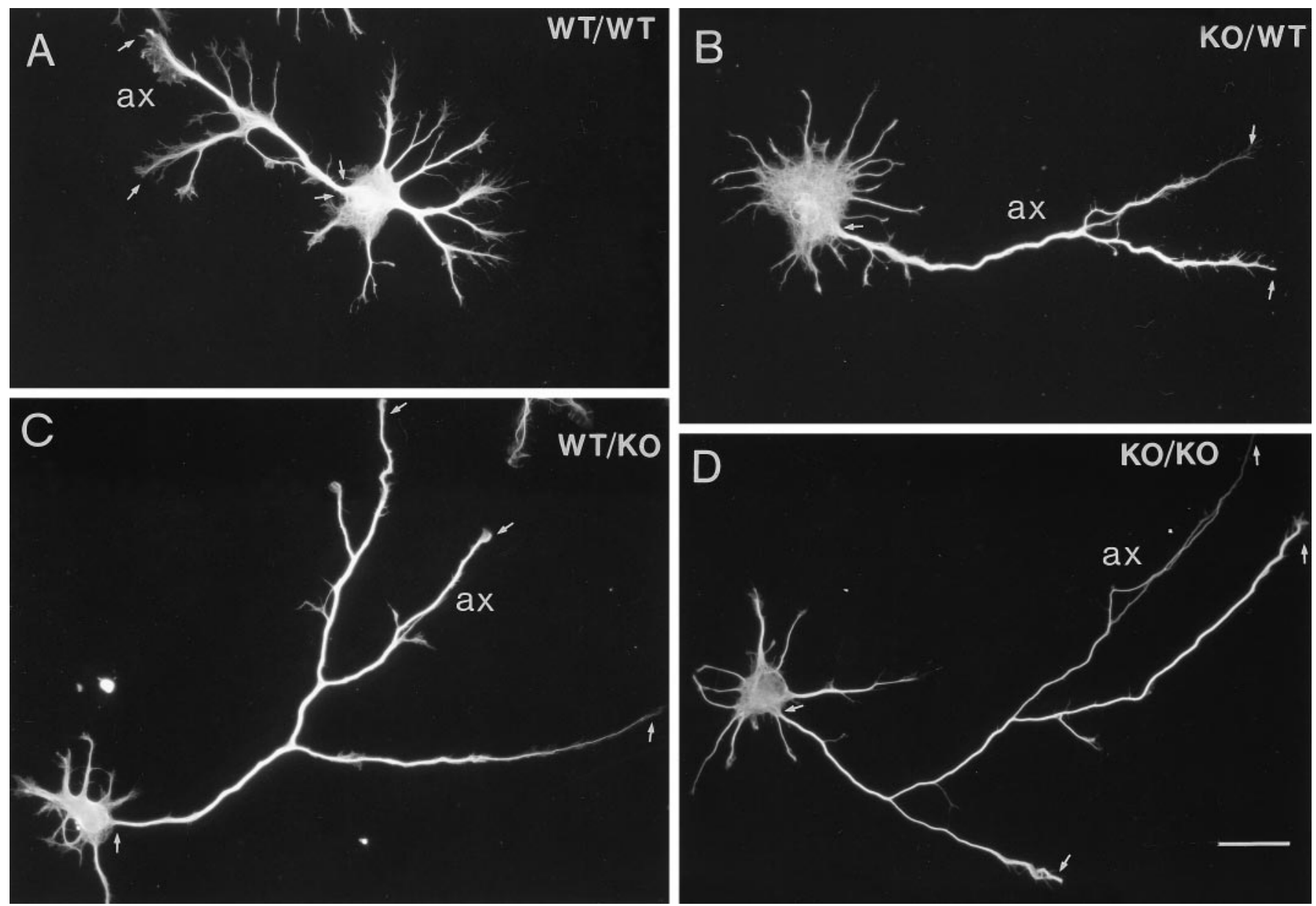

Figure 4. Hippocampal neurons cocultured with wild-type and $\beta$ PP-deficient astrocytes for $3 \mathrm{~d}$. Neurons had extensive axon (ax) and minor process development (small processes emanating from the cell body) by $3 \mathrm{~d}$ in vitro. Proximal and distal portions of each axon are indicated by small arrows for a wild-type neuron cocultured with wild-type astrocytes $(A)$, a $\beta$ PP-deficient neuron cocultured with wild-type astrocytes $(B)$, a wild-type neuron cocultured with $\beta$ PP-deficient astrocytes $(C)$, and a $\beta$ PP-deficient neuron cocultured with $\beta$ PP-deficient astrocytes $(D)$. The developing predendritic, minor processes are more pronounced for neurons cultured with wild-type astrocytes for $3 \mathrm{~d}(A, B)$ than for neurons grown with $\beta$ PP-deficient glia $(C, D)$. WT/WT, Wild-type neurons cocultured with wild-type astrocytes; $K O / W T, \beta$ PP-deficient neurons cocultured with wild-type astrocytes; $W T / K O$, wild-type neurons cocultured with $\beta$ PP-deficient knock-out astrocytes; $K O / K O, \beta P P$-deficient neurons cocultured with $\beta$ PP-deficient astrocytes. Scale bar, $25 \mu \mathrm{m}$.
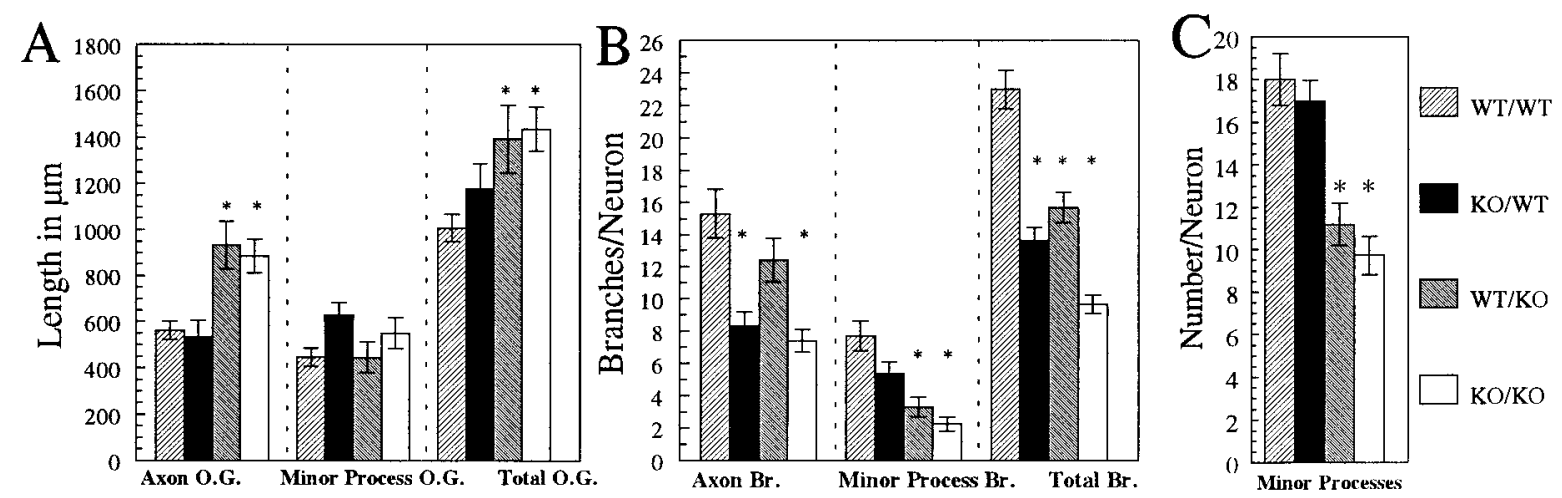

Figure 5. $\quad \beta$ PP-related effects on hippocampal neurons grown in coculture with wild-type and $\beta$ PP-deficient astrocytes for $3 \mathrm{~d}$. $A$, Axon outgrowth was significantly less for neurons cocultured with wild-type astrocytes, which secrete $\beta \mathrm{PP}_{\mathrm{s}}$ and $\mathrm{A} \beta$. Minor process outgrowth was not significantly different for the four coculture conditions. Total outgrowth, which paralleled axon outgrowth, was greater in the absence of $\beta$ PP secretory products. $B$, Wild-type neurons, which express cell-surface $\beta \mathrm{PP}$, had more axon branching than $\beta \mathrm{PP}$-deficient neurons in both coculture conditions. Minor process branching was enhanced for neurons grown with wild-type astrocytes, which secrete $\beta \mathrm{PP}_{\mathrm{s}}$ and $\mathrm{A} \beta$. Total branching was enhanced for wild-type neurons cocultured with wild-type astrocytes $(W T / W T)$. $C$, More minor processes were produced by neurons cocultured with wild-type astrocytes, which secrete $\beta$ PP and $_{\mathrm{s}}$ $\mathrm{A} \beta . W T / W T$, Wild-type neurons cocultured with wild-type astrocytes; $K O / W T, \beta$ PP-deficient knock-out neurons with wild-type astrocytes; $W T / K O$, wild-type neurons with $\beta$ PP-deficient knock-out astrocytes; $K O / K O, \beta P P$-deficient knock-out neurons with $\beta P P$-deficient knock-out astrocytes; $O . G$., outgrowth; $B r$., branches. Data are averages of 40 neurons \pm SEM. *Significantly different from WT/WT cocultures. Histogram legend in $C$ applies to $A-C$. 
growth that was associated with significantly shorter axons at $1 \mathrm{~d}$ (Fig. $3 A$ ). Minor process outgrowth was similar for $\beta$ PP-deficient neurons and wild-type neurons cocultured with wild-type astrocytes (Fig. $3 A$ ); however, $\beta$ PP-deficient neurons cocultured with $\beta$ PP-deficient astrocytes had significantly diminished minor process outgrowth (Fig. $3 A$ ). It is noteworthy that a marked reduction in minor process outgrowth was observed for both wild-type and $\beta \mathrm{PP}$-deficient neurons when they were cocultured with $\beta \mathrm{PP}$ deficient astrocytes for $1 \mathrm{~d}$ (Fig. $3 A$ ).

Axon branching was less for $\beta \mathrm{PP}$-deficient neurons cocultured with wild-type astrocytes and somewhat reduced for $\beta P P$ deficient neurons cocultured with $\beta P P$-deficient astrocytes at $1 \mathrm{~d}$ (Fig. $3 B$ ). Minor processes were affected similarly. Minor process branching for $\beta \mathrm{PP}$-deficient neurons in both coculture conditions was less than that observed for wild-type neurons cocultured with wild-type astrocytes (Fig. $3 B$ ). Furthermore, although $\beta P P$ deficient neurons cocultured with $\beta$ PP-deficient astrocytes had significantly more minor processes at $1 \mathrm{~d}$ (Fig. $3 C$ ), these minor processes were significantly shorter than those of wild-type neurons cocultured with wild-type astrocytes (Fig. $3 A$ ).

\section{Hippocampal neurons cocultured with wild-type astrocytes for $3 \mathrm{~d}$ have shorter axons but enhanced minor process outgrowth}

By $3 \mathrm{~d}$ in culture wild-type neurons cocultured with wild-type astrocytes (Fig. $4 A$ ) and wild-type neurons cocultured with $\beta \mathrm{PP}$ deficient astrocytes (Fig. $4 C$ ) had grown considerably. However, the distribution of outgrowth was markedly different, depending on the astrocytes used for coculture. Interestingly, axon outgrowth from wild-type neurons, which had been significantly greater in the presence of wild-type astrocytes at $1 \mathrm{~d}$, was now greater in the presence of $\beta \mathrm{PP}$-deficient astrocytes at $3 \mathrm{~d}$ (Fig. $5 A)$. Similarly, $\beta \mathrm{PP}$-deficient neurons cocultured with $\beta \mathrm{PP}$ deficient astrocytes also had significantly longer axons, as compared with $\beta$ PP-deficient neurons cocultured with wild-type astrocytes at $3 \mathrm{~d}$ (Fig. $5 A$ ). Therefore, the growth of both wild-type and $\beta$ PP-deficient neurons with wild-type astrocytes was found to limit the growth of axons. Both wild-type and $\beta$ PP-deficient neurons had significantly longer axons and greater total outgrowth when grown with $\beta$ PP-deficient astrocytes for $3 \mathrm{~d}$ (Fig. $5 A)$. Although somewhat greater for $\beta \mathrm{PP}$-deficient neurons than for wild-type neurons, minor process outgrowth was not significantly different for neurons in the four coculture conditions by $3 \mathrm{~d}$ (Fig. 5A).

Total branching at $3 \mathrm{~d}$ was greatest for wild-type neurons cocultured with wild-type astrocytes (Fig. 4A) than for neurons in the other three coculture conditions (Figs. 4, 5B). Axon branching was significantly greater for wild-type neurons than for $\beta \mathrm{PP}$ deficient neurons in both astrocyte coculture conditions (Fig. $5 B$ ). Minor process branching was greater for all neurons grown in the presence of wild-type astrocytes for $3 \mathrm{~d}$, with wild-type neurons having the most minor process branching of the four coculture conditions (Fig. $5 B$ ). Growth of both wild-type and $\beta$ PP-deficient neurons with $\beta \mathrm{PP}$-deficient astrocytes produced less minor process branching. Although minor process branching had nearly doubled for neurons grown with wild-type astrocytes for $3 \mathrm{~d}$, neurons cocultured with $\beta$ PP-deficient astrocytes had markedly less minor process branching by $3 \mathrm{~d}$ (compare Figs. $3 B$ and $5 B$ ). Minor process branching for wild-type neurons was increased only slightly at $3 \mathrm{~d}$ relative to $1 \mathrm{~d}$, and minor process branching was actually decreased by $3 \mathrm{~d}$ for $\beta \mathrm{PP}$-deficient neurons (compare Figs. $3 B$ and $5 B$ ). Of all the measures obtained in these studies, minor process branching for $\beta \mathrm{PP}$-deficient neurons cocultured with $\beta$ PP-deficient astrocytes was the only value that did not increase between 1 and 3 d. Minor process numbers were increased significantly for neurons cocultured in the presence of wild-type astrocytes but only slightly increased for neurons grown with $\beta$ PP-deficient astrocytes between 1 and 3 d (compare Figs. $3 C$ and $5 C$ ).

\section{DISCUSSION}

Hippocampal neurons from $\beta$ PP-deficient transgenic knock-out mice and wild-type control animals were analyzed for survival and neurite outgrowth. Neurons express abundant $\beta P P$, yet relatively little $\beta \mathrm{PP}$ is processed into secreted products by neurons (Hung et al., 1992; Wertkin et al., 1993; LeBlanc et al., 1996), implying that a larger percentage of neuronal $\beta$ PP remains intact. Although many biological functions have been attributed to $\beta \mathrm{PP}_{\mathrm{s}}$ (Saitoh et al., 1989; Schubert et al., 1989; Mattson et al., 1993; Goodman et al., 1994; Jin et al., 1994; Mattson, 1994; Yamamoto et al., 1994), much less is known about uncleaved $\beta$ PP function. Full-length $\beta \mathrm{PP}$ is localized at hippocampal neuron cell surfaces (Yamazaki et al., 1995) and can mediate binding to other cell surfaces to enhance adhesion and neurite outgrowth (Qiu et al., 1995). To directly evaluate a role for cell-associated $\beta P P$, we measured differences in neuron development attributable to cellassociated $\beta \mathrm{PP}$ present on wild-type neurons, but not on $\beta \mathrm{PP}$ deficient neurons. In addition, we evaluated the responses of neurons to $\beta \mathrm{PP}$ secreted products by growing hippocampal neurons with wild-type astrocytes that release $\beta \mathrm{PP}_{\mathrm{s}}$ and $\mathrm{A} \beta$ (LeBlanc et al., 1996). Our data have generated two major findings previously unreported for $\beta \mathrm{PP}$ : (1) that cell-associated $\beta \mathrm{PP}$ plays a role in neuron survival in culture and (2) that both cell-associated $\beta \mathrm{PP}$ and $\beta \mathrm{PP}$ secreted products contribute to axon and dendritic outgrowth and arborization in a complex manner.

Because $\beta$ PP plays a role in neuronal cell adhesion (Schubert et al., 1989; LeBlanc et al., 1992; Qiu et al., 1995), we tested $\beta \mathrm{PP}$-deficient neurons for attachment and spreading. The lack of $\beta \mathrm{PP}$ expression by $\beta \mathrm{PP}$-deficient neurons did not affect attachment or spreading on poly-L-lysine-treated substrates. This result is not surprising because neurons adhere better to poly-L-lysinetreated substrates than to tissue culture plastic, fibronectin, or laminin, even in the presence of anti- $\beta$ PP antibodies (Breen et al., 1991). Furthermore, neurons have many alternate adhesion molecules (e.g., NCAM, L1, and Axonin-1; for review, see Fields and Itoh, 1996) that effectively mediate attachment.

Although $\beta \mathrm{PP}_{\mathrm{s}}$ has been proposed to contribute to cell survival (Yamamoto et al., 1995), ours are the first data to show that endogenous cell-associated $\beta$ PP contributes to neuron survival. In both B27-supplemented media and in neuron/astrocyte cocultures there was a significantly greater loss of $\beta \mathrm{PP}$-deficient neurons, as compared with wild-type neurons. Moreover, $\beta \mathrm{PP}$ deficient hippocampal cultures had the identical degree of neuron loss whether grown with wild-type or $\beta \mathrm{PP}$-deficient astrocytes (Fig. 1C). Culturing $\beta \mathrm{PP}$-deficient neurons in the presence of wild-type astrocyte-conditioned medium (which includes $\beta \mathrm{PP}_{\mathrm{s}}$ and $\mathrm{A} \beta$ ) had no rescuing effect on the $\beta \mathrm{PP}$-deficient neurons that died during the $3 \mathrm{~d}$ of culture. This observation strongly implicates cell-associated $\beta \mathrm{PP}$ in survival of the cultured neurons. Two potential modes of action for cell-associated $\beta \mathrm{PP}$ in neuron survival include (1) a direct promotion of neuron survival (as described below) or (2) a homophilic interaction between $\beta \mathrm{PP}_{\mathrm{s}}$ or $\mathrm{A} \beta$ and cell-associated $\beta \mathrm{PP}$, now absent from $\beta \mathrm{PP}$-deficient neurons. Thus $\beta$ PP-deficient neurons have provided the first evidence 
that endogenous cell-associated $\beta P P$ directly contributes to neuron survival.

The influence of $\beta P P$ on the development of axonal and dendritic processes is more complex than its effects on neuronal viability described above. Our results showed that both cellular $\beta \mathrm{PP}$ and $\beta \mathrm{PP}$ secreted products contributed to all aspects of neurite outgrowth examined in this study. First, $\beta P P$ appears to play a role in axon development. This is suggested by $\beta$ PPdeficient neurons having markedly shorter axons at $1 \mathrm{~d}$. Because $\beta \mathrm{PP}$-deficient neurons grown with wild-type astrocytes ultimately developed axons equal in length to those of wild-type neurons, it appears that cell-associated $\beta \mathrm{PP}$ primarily contributes to the onset of axon formation. Neither minor process outgrowth nor total outgrowth was affected significantly by the absence of $\beta \mathrm{PP}$ from $\beta$ PP-deficient neurons grown with wild-type astrocytes, suggesting a specific effect on axon development. Although axons are shorter at $1 \mathrm{~d}$ in the absence of neuronal $\beta \mathrm{PP}$ expression, the manner in which this occurs is undetermined. Perhaps in the absence of cell-associated $\beta P P$, neurons have difficulty determining which process will become the axon.

Second, cell-associated $\beta \mathrm{PP}$ also contributes to arborization and process formation. Wild-type neurons always had more branching and ultimately had more minor processes than did $\beta$ PP-deficient hippocampal neurons. Again, the most pronounced effect for cell-associated $\beta \mathrm{PP}$ on branching was observed for axons. Because $\beta$ PP-deficient neurons had axons that were initially shorter and always less branched, the data suggest that cell-associated $\beta$ PP contributes to normal axon formation. Dendritic (i.e., minor process) branching also was affected by the absence of $\beta P P$. The finding that all processes branched less when neurons lacked $\beta P P$ suggests that axon/dendritic connectivity may be abnormal in the $\beta$ PP-deficient mice and may underlie the diminished motor functions observed in these animals (Zheng et al., 1995). Indeed, $\beta$ PP is abundantly expressed by motor neurons during normal mouse development (Salbaum and Ruddle, 1994).

Taken together, cell-associated neuronal $\beta \mathrm{PP}$ appears to function both in neuronal survival and neurite outgrowth. The mechanisms by which $\beta$ PP promotes these effects are unclear. Nonetheless, it is interesting to hypothesize that cell-surface $\beta \mathrm{PP}$ specifically mediates these effects. Cell-surface $\beta \mathrm{PP}$ appears to use the region between amino acids $444-592$ ( $\beta \mathrm{PP}_{695}$ numbering) for cell adhesion, neurite outgrowth (Qiu et al., 1995), and protection against excitotoxicity (Mattson, 1994). Cell-surface $\beta P P$ may transduce signals important for neuron growth and survival by an interaction of its $C$ terminus with the heterotrimeric $\mathrm{G}_{0^{-}}$ protein (Okamoto, 1995). $\beta$ PP also may provide a molecular link between the neuronal cytoskeleton (Allinquant et al., 1994) and the extracellular environment and thereby contribute to neurite outgrowth and pathfinding. The recent observation of colocalization of cell-surface $\beta \mathrm{PP}$ with integrins in neural cells (Storey et al., 1996; Yamazaki et al., 1997) is consistent with this hypothesis. Although $\beta$ PP expression may be most important for particular subsets of neurons (Salbaum and Ruddle, 1994) or during specific neurodevelopmental stages, its significance is substantiated nonetheless by the finding that $\beta \mathrm{PP}$-deficient neurons survived less well in culture. Therefore, our results confirm the importance of $\beta \mathrm{PP}$ to neuron function and provide compelling evidence that cell-associated, possibly cell-surface $\beta \mathrm{PP}$ mediates neuron survival.

The effects of $\beta P P$ secretory products were determined by growing hippocampal neurons with astrocytes from wild-type and $\beta$ PP-deficient mice. Although an earlier study suggested that neural cells secreted little $\beta \mathrm{PP}_{\mathrm{s}}$ (Haass et al., 1991), a recent report shows that up to $40 \%$ of total astrocytic $\beta \mathrm{PP}$ is processed into $\beta \mathrm{PP}_{\mathrm{s}}$ but very little into $\mathrm{A} \beta$ (LeBlanc et al., 1996). By $3 \mathrm{~d}$ in vitro, wild-type and $\beta \mathrm{PP}$-deficient neurons cocultured with wildtype astrocytes had significantly shorter axons than neurons cocultured with $\beta \mathrm{PP}$-deficient astrocytes. It is noteworthy that both wild-type and $\beta \mathrm{PP}$-deficient hippocampal neurons showed the identical axonal response to both wild-type and $\beta$ PP-deficient astrocyte conditioned media. Unlike the diminution of axon outgrowth, the number of minor processes and the number of branch points were significantly greater for neurons cultured with wildtype astrocytes. These results suggest that soluble factors present in wild-type astrocyte conditioned medium modulate axon growth and process branching. Neurite growth can be affected by both $\beta \mathrm{PP}_{\mathrm{s}}$ (Mattson, 1994) and $\mathrm{A} \beta$ (Koo et al., 1993). Dendritic growth and branching in our studies are similar to the effect described by Mattson, using exogenous $\beta \mathrm{PP}_{\mathrm{s}}$ (1994); however, we did not assess the effect of $\beta \mathrm{PP}_{\mathrm{s}}$ or $\mathrm{A} \beta$ on neurite development directly. In addition, other secreted factors besides $\beta \mathrm{PP}_{\mathrm{s}}$ or $\mathrm{A} \beta$ may have been present or absent from $\beta \mathrm{PP}$-deficient astrocyte conditioned medium. Alternately, a complexing of $\beta \mathrm{PP}_{\mathrm{s}}$ with other cell-surface proteins as described for heparan sulfate proteoglycan (Small et al., 1994) or A $\beta$ interacting with neuronal adhesion molecules (Sabo et al., 1995) also may have contributed to the growth differences observed for neurons cocultured with wild-type astrocytes. Further analysis is required to define the mechanism underlying our observations.

In summary, the data indicate that both cell-associated $\beta \mathrm{PP}$ and secreted $\beta \mathrm{PP}$ products appear to be important for normal neuronal development. Cell-associated $\beta \mathrm{PP}$, possibly cell-surface $\beta \mathrm{PP}$, enhances neuron survival, the timely initiation of axon growth, and axon arborization. $\beta$ PP secreted products contribute to axonal and dendritic growth in a manner that modulates neuronal polarity and appears to limit the growth of axons at the same time dendritic growth is enhanced and may be involved in coordinating the timing of connections in the developing CNS. Future analyses with this rodent model should help to elucidate the complex role of $\beta \mathrm{PP}$ in both the developing and mature CNS.

\section{REFERENCES}

Allinquant B, Moya KL, Bouillot C, Prochiantz A (1994) Amyloid precursor protein in cortical neurons: coexistence of two pools differentially distributed in axons and dendrites and association with cytoskeleton. J Neurosci 14:6842-6854.

Allinquant B, Hantraye P, Mailleux P, Moya K, Bouillot C, Prochiantz A (1995) Downregulation of amyloid precursor protein inhibits neurite outgrowth in vitro. J Cell Biol 128:919-927.

Banker G, Goslin K (1991) Rat hippocampal neurons in low-density culture. In: Culturing nerve cells (Banker G, Goslin K, eds), pp 251281. Cambridge, MA: MIT.

Bottenstein JE, Sato GH (1979) Growth of a rat neuroblastoma cell line in serum-free supplemented medium. Proc Natl Acad Sci USA 76:514-517.

Brandt R, Lee G (1993) Functional organization of microtubuleassociated protein tau. J Biol Chem 268:3414-3419.

Breen K, Bruce M, Anderson B (1991) $\beta$-Amyloid precursor protein mediates neuronal cell-cell and cell-surface adhesion. J Neurosci Res 28:90-100.

Caceres A, Banker GA, Steward O, Binder L, Payne M (1984) MAP2 is localized to the dendrites of hippocampal neurons which develop in culture. Dev Brain Res 13:314-318.

Canoll PD, Musacchio JM, Hardy R, Reynolds R, Marchionni MA, Salzer JL (1996) GGF/neuregulin is a neuronal signal that promotes the proliferation and survival and inhibits the differentiation of oligodendrocyte progenitors. Neuron 17:229-243.

Chin L-S, Lian L, Ferreira A, Kosik K, Greengard P (1995) Impairment 
of axonal development and of synaptogenesis in hippocampal neurons of synapsin I-deficient mice. Proc Natl Acad Sci USA 92:9230-9234.

Dotti CG, Sullivan CA, Banker GA (1988) The establishment of polarity by hippocampal neurons in culture. J Neurosci 8:1454-1468.

Ferreira A, Caceres A (1992) Expression of the class III $\beta$-tubulin isotype in developing neurons in culture. J Neurosci Res 32:516-529.

Ferreira A, Caceres A, Kosik KS (1993) Intraneuronal compartments of the amyloid precursor protein. J Neurosci 13:3112-3123.

Fields RD, Itoh K (1996) Neural cell adhesion molecules in activitydependent development and synaptic plasticity. Trends Neurosci 19:473-480.

Frankfurter A, Binder LI, Rebhun L (1986) Limited tissue distribution of a novel $\beta$-tubulin isoform. J Cell Biol 103:273.

Goodman Y, Mattson MP (1994) Secreted forms of $\beta$-amyloid precursor protein protect hippocampal neurons against amyloid $\beta$-peptideinduced oxidative injury. Exp Neurol 128:1-12.

Haass C, Hung AY, Selkoe DJ (1991) Processing of $\beta$-amyloid precursor protein in microglia and astrocytes favors an internal localization over constitutive secretion. J Neurosci 11:3783-3793.

Hung AY, Koo EH, Haass C, Selkoe DJ (1992) Increased expression of $\beta$-amyloid precursor protein during neuronal differentiation is not accompanied by secretory cleavage. Proc Natl Acad Sci USA 89:9439-9443.

Jin L-W, Ninomiya H, Roch J-M, Schubert D, Masliah E, Otero DA, Saitoh T (1994) Peptides containing the RERMS sequence of amyloid $\beta$-protein precursor bind cell surface and promote neurite extension. J Neurosci 14:5461-5470.

Kang J, Lemaire H, Unterbeck A, Salbaum JM, Masters CL, Grzeschik K, Multhaup G, Beyreuther K, Muller-Hill B (1987) The precursor of Alzheimer's disease amyloid A4 protein resembles a cell-surface receptor. Nature 325:733-736.

Koo EH, Park L, Selkoe DJ (1993) Amyloid $\beta$-protein as a substrate interacts with extracellular matrix to promote neurite outgrowth. Proc Natl Acad Sci USA 90:4748-4752.

LeBlanc AC, Kovacs DM, Chen HY, Villare F, Tykocinski M, AutilioGambetti L, Gambetti P (1992) Role of amyloid precursor protein (APP): study with antisense transfection of human neuroblastoma cells. J Neurosci Res 31:635-645.

LeBlanc AC, Xue R, Gambetti P (1996) Amyloid precursor protein metabolism in primary cell cultures of neurons, astrocytes, and microglia. J Neurochem 66:2300-2310.

Majocha RE, Agrawal S, Tang J-Y, Humke EW, Marotta CA (1994) Modulation of the PC12 cell response to nerve growth factor by antisense oligonucleotide to amyloid precursor protein. Cell Mol Neurobiol 14:425-437.

Mattson MP (1994) Secreted forms of $\beta$-amyloid precursor protein modulate dendrite outgrowth and calcium responses to glutamate in cultured embryonic hippocampal neurons. J Neurobiol 25:439-450.

Mattson MP, Cheng B, Culwell A, Esch F, Lieberberg I, Rydel R (1993) Evidence for excitoprotective and intraneuronal calcium-regulating roles for secreted forms of the $\beta$-amyloid precursor protein. Neuron 10:243-254.

Milward EA, Papadopoulos R, Fuller SJ, Moir RD, Small D, Beyreuther K, Masters CL (1992) The amyloid protein precursor of Alzheimer's disease is a mediator of the effects of nerve growth factor on neurite outgrowth. Neuron 9:129-137.

Moya KL, Benowitz LI, Schneider GE, Allinquant B (1994) The amyloid precursor protein is developmentally regulated and correlated with synaptogenesis. Dev Biol 161:597-603.

Okamoto T, Takeda S, Murayama Y, Ogata E, Nishimoto I (1995)
Ligand-dependent G-protein coupling function of amyloid transmembrane precursor. J Biol Chem 270:4205-4208.

Qiu WQ, Ferreira A, Miller C, Koo EH, Selkoe DJ (1995) Cell-surface $\beta$-amyloid precursor protein stimulates neurite outgrowth of hippocampal neurons in an isoform-dependent manner. J Neurosci 15:2157-2167.

Sabo S, Lambert MP, Kessey K, Wade W, Krafft G, Klein WL (1995) Interaction of $\beta$-amyloid peptides with integrins in a human nerve cell line. Neurosci Lett 184:25-28.

Saitoh T, Sundsmo M, Roch J-M, Kimura N, Cole G, Schenk D (1989) Secreted form of amyloid $\beta$ protein precursor is involved in the growth regulation of fibroblasts. Cell 58:615-622.

Salbaum MJ, Ruddle FH (1994) Embryonic expression pattern of amyloid protein precursor suggests a role in differentiation of specific subsets of neurons. J Exp Zool 269:116-127.

Schubert D, Jin L-W, Saitoh T, Cole G (1989) The regulation of amyloid $\beta$ protein precursor secretion and its modulatory role in cell adhesion. Neuron 3:689-694.

Selkoe DJ (1994) Alzheimer's disease: a central role for amyloid. J Neuropathol Exp Neurol 53:438-447.

Shivers BD, Hilbich C, Multhaup G, Salbaum M, Beyreuther K, Seeburg PH (1988) Alzheimer's disease amyloidogenic glycoprotein: expression pattern in rat brain suggests a role in cell contact. EMBO J 7:1365-1370.

Small DH, Nurcombe V, Reed G, Clarris H, Moir R, Beyreuther K, Master CL (1994) A heparin-binding domain in the amyloid protein precursor of Alzheimer's disease is involved in the regulation of neurite outgrowth. J Neurosci 14:2117-2127.

Storey E, Beyreuther K, Masters CL (1996) Alzheimer's disease amyloid precursor protein on the surface of cortical neurons in primary culture co-localizes with adhesion patch components. Brain Res 735:217-231.

Tawil N, Wilson P, Carbonetto S (1993) Integrins in point contacts mediate cell spreading: factors that regulate integrin accumulation in point contacts vs focal contacts. J Cell Biol 120:261-271.

Wertkin AM, Turner RS, Pleasure SJ, Golde TE, Younkin SG, Trojanowski JQ, Lee VM-Y (1993) Human neurons derived from a teratocarcinoma cell line express solely the 695-amino acid amyloid precursor protein and produce intracellular $\beta$-amyloid or A4 peptides. Proc Natl Acad Sci USA 90:9513-9517.

Whitson JS, Selkoe DJ, Cotman CW (1989) Amyloid beta protein enhances the survival of hippocampal neurons in vitro. Science 243:1488-1490.

Yamamoto K, Miyoshi T, Yae T, Kawashima K, Araki H, Hanad K, Otero DA, Roch JM, Saitoh T (1994) The survival of rat cerebral cortical neurons in the presence of trophic APP peptides. J Neurobiol 25:585-594.

Yamazaki T, Selkoe DJ, Koo EH (1995) Trafficking of cell surface $\beta$-amyloid precursor protein: retrograde and transcytotic transport in cultured neurons. J Cell Biol 129:431-442.

Yamazaki T, Koo EH, Selkoe DJ (1997) Cell surface amyloid $\beta$-protein precursor colocalizes with $\beta 1$ integrins at substrate contact sites in neural cells. J Neurosci 17:1004-1010.

Yankner BA (1996) Mechanisms of neuronal degeneration in Alzheimer's disease. Neuron 16:921-932.

Yankner BA, Duffy LK, Kirschner DA (1990) Neurotrophic and neurotoxic effects of amyloid beta protein: reversal by tachykinin neuropeptides. Science 250:279-282.

Zheng H, Jiang M, Trumbauer ME, Sirinathsinghji DJ, Hopkins R, Smith DW, Heavens RP, Dawson GR, Boyce S, Conner MW, Sisodia S, Van der Ploeg L (1995) $\beta$-Amyloid precursor protein-deficient mice show reactive gliosis and decreased locomotor activity. Cell 81:525-531. 